\title{
The absolute maximum and detailed phenomenology of the muon magnetic moment in the 2HDM
}

\section{Adriano Cherchiglia}

Universidade Federal do ABC - Centro de Ciências Naturais e Humanas, Santo André - Brazil

\section{Dominik Stöckinger}

Institut für Kern- und Teilchenphysik, TU Dresden, 01069 Dresden, Germany

\section{Hyejung Stöckinger-Kim*†}

Institut für Kern- und Teilchenphysik, TU Dresden, 01069 Dresden, Germany

E-mail: hyejung.stoeckinger-kimetu-dresden.de

\begin{abstract}
We present the muon magnetic moment $g-2$ in the flavour-aligned 2HDM evaluated by employing the recent result of full two-loop computation and making comprehensive use of experimental constraints from Higgs and flavour physics and characterize the parameter regions possible to explain the current $3 \sigma$ deviation. We particularly focus on the light CP-odd neutral Higgs boson $A$ and present the maximum possible Yukawa couplings to leptons and quarks of a light $A$ allowed by the LHC and $B$-physics results, which can enhance $a_{\mu}^{2 \mathrm{HDM}}$ in this mass region. As a result we find an overall maximum of $45 \times 10^{10}$ for $a_{\mu}^{2 \mathrm{HDM}}$ in the parameter region $20<M_{A}<100 \mathrm{GeV}$.
\end{abstract}

The 39th International Conference on High Energy Physics (ICHEP2018)

4-11 July, 2018

Seoul, Korea

${ }^{*}$ Speaker.

†We acknowledge financial support from DFG Grant STO/876/6-1, and CAPES (Coordenação de Aperfeiçoamento de Pessoal de Nível Superior), Brazil. The work has further been supported by the high-performance computing cluster Taurus at ZIH, TU Dresden, and by the National Science Center, Poland, through the HARMONIA project under contract UMO-2015/18/M/ST2/00518 (2016-2019). 


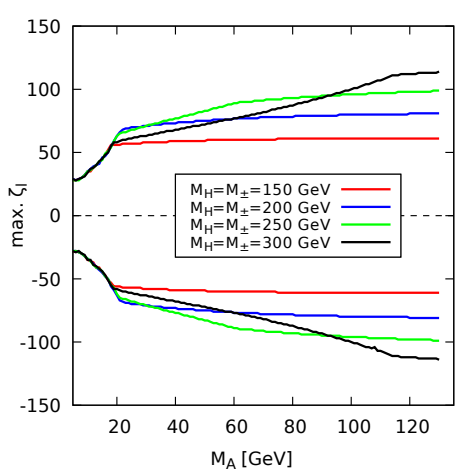

(a)

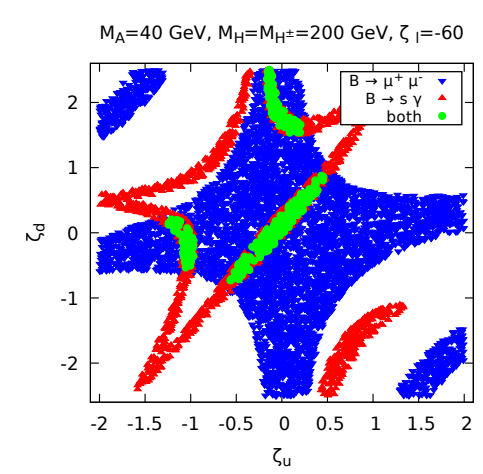

(b)

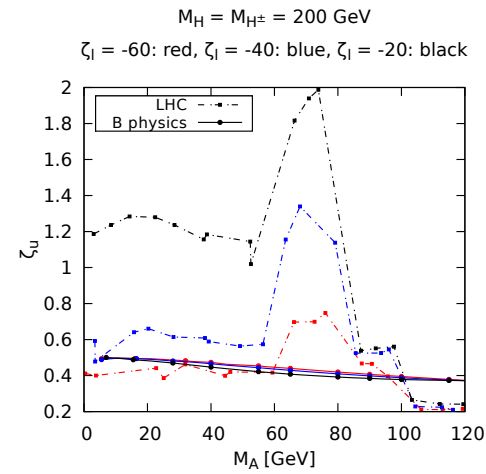

(c)

Figure 1

\section{Introduction}

The present proceedings are a review of the detailed numerical analysis of the muon magnetic moment $g-2$ in the two-Higgs doublet model (2HDM) in Ref. [1], which is obtained by using the complete two-loop correction results in Ref. [2].

The discrepancy in the muon magnetic moment $g-2$ between the experimental measurement [3] and the Standard Model (SM) predictions evaluated from the most recent studies [4-6] amounts to

$$
a_{\mu}^{\text {Exp. }}-a_{\mu}^{\mathrm{SM}}=\left(28.1 \pm 6.3^{\text {Exp. }} \pm 3.6^{\mathrm{Th}}\right) \times 10^{-10} .
$$

A substantial increase in the accuracy is expected in the further measurements at Fermilab and J-PARC. This persisting 3-4 $\sigma$ deviation motivates new physics (NP) scenarios beyond the SM. Typically the NP contributions to the muon $g-2$ is suppressed by heavy NP masses $\sim\left(m_{\mu}^{2} / M_{\mathrm{NP}}^{2}\right)$. Therefore, a NP scenario with a very high $M_{\mathrm{NP}}$ scale is inappropriate to explain the discrepancy in Eq. 1.1.

The 2HDM is a minimal extension to the SM and includes four physical Higgs masses $M_{h}, M_{H}$, $M_{A}$ and $M_{H^{ \pm}}$. The two neutral CP-even Higgs mass eigenstates $h$ and $H$ are combined states of the two scalar doublets with mixing angle $\sin (\beta-\alpha)$. When $\sin (\beta-\alpha)=1$ the SM-limit is reached and hence, $h$ becomes an SM-like Higgs boson $h_{\mathrm{SM}}$. The 2HDM muon $g-2$ contribution $a_{\mu}^{2 \mathrm{HDM}}$ is obtained from the additional Higgs bosons: $A, H, H^{ \pm} . Z_{2}$ symmetry permits four different Yukawa coupling scenarios: the usual type I, II, X and Y. In the more general flavour-aligned $2 \mathrm{HDM}$ (A2HDM) [7] the Yukawa couplings are proportional to the Yukawa parameters $\zeta_{l, u, d}$.

Here we concentrate on three questions: (a) What are the constraints on the 2HDM parameters relevant for $a_{\mu}$ ? (b) What are the parameter regions to explain the current deviation in $a_{\mu}$ ? (c) What is the overall maximum possible value of $a_{\mu}$ in the 2HDM?

\section{The $2 H D M$ contributions and constraints on the parameters}

As the one-loop contributions are suppressed by higher order of muon mass $\sim \mathscr{O}\left(m_{\mu}^{4}\right)$ through the Yukawa couplings the leading contributions to $a_{\mu}^{2 \mathrm{HDM}}$ come from the Barr-Zee type two-loop 
diagrams, where the muon line is coupled with one gauge boson and one Higgs boson with either fermions or bosons in the Barr-Zee loop. The leading fermion loop corrections are from the BarrZee $\tau$ - or $t$-loop corrections. The following semi-numerical expressions for the leading fermionic two-loop and bosonic two-loop contributions are useful approximations to estimate the contributions for light $M_{A}$ :

$$
\begin{aligned}
a_{\mu}{ }^{\tau-\text { loop, } 2 \mathrm{HDM}} & \simeq\left(\frac{\zeta_{l}}{100}\right)^{2}\left\{\frac{8+4 \hat{x}_{M_{A}}^{2}+2 \ln \left(\hat{x}_{M_{A}}\right)}{\hat{x}_{M_{A}}^{2}}\right\}, \\
a_{\mu}{ }^{t-\text { loop, } 2 \mathrm{HDM}} & \simeq\left(\frac{-\zeta_{l} \zeta_{u}}{100}\right)\left\{54-14 \ln \left(\hat{x}_{M_{A}}\right)-15 \ln \left(\hat{x}_{M_{H}}\right)\right\}, \\
\left|a_{\mu}^{B 2 \mathrm{HDM}}\right| & \simeq \rho\left|C_{H H^{+} H^{-}} / \mathrm{GeV}\right|\left|\zeta_{l}\right| \times 10^{-15},
\end{aligned}
$$

where $\rho=6,3,2,1$ for $M_{H}=M_{H^{ \pm}}=150,200,250,300 \mathrm{GeV}$ respectively.

The $\tau$-loop correction is enhanced by $\zeta_{l}^{2}$ and the $t$-loop correction by $\zeta_{l} \zeta_{u}$. Thus the 2HDM fermion loop contribution $a_{\mu}^{F}{ }^{2 \mathrm{HDM}}$ depends on the Yukawa parameters $\zeta_{l}$ and $\zeta_{u}$ as well as the Higgs boson masses. The $t$-loop correction becomes positive when we set the signs of $\zeta_{l}$ and $\zeta_{u}$ opposite to each other. In the numerical analysis we set $\zeta_{l}$ negative and $\zeta_{u}$ positive. Eq. 2.3 shows that the bosonic contributions are enhanced by the triple Higgs coupling constant $C_{\mathrm{HH}^{+} \mathrm{H}^{-}}$and are proportional to $\zeta_{l}$. From Eqs. 2.1- 2.3 we find that $\zeta_{l}, \zeta_{u}$ and $M_{A}$ are the determining parameters for $a_{\mu}^{2 \mathrm{HDM}}$.

It is necessary to investigate the allowed $\zeta_{l}$ and $\zeta_{u}$ for given Higg boson mass points to analyse

$a_{\mu}^{2 \mathrm{HDM}}$. $\zeta_{l}$ is constrained by $\tau$-decay, leptonic $Z$-decay and $e e \rightarrow \tau \tau A$ searches at LEP. Theses experiments set upper bounds on $\zeta_{l}$. As the decay rates in the $2 \mathrm{HDM}$ are enhanced by $\zeta_{l}$, large $\zeta_{l}$ leads to disagreement with experimental observations. Fig. 1a shows the upper bounds on $\zeta_{l}$ for different Higgs boson mass values. For $20<M_{A}<120 \mathrm{GeV}$ and $150<M_{H}\left(=M_{H^{ \pm}}\right)<300 \mathrm{GeV}$ we obtain $\left|\zeta_{l}\right|<60$.

$\zeta_{u}$ gains constraints from $B$-decay channels $\left(b \rightarrow s \gamma\right.$ and $\left.B_{s} \rightarrow \mu \mu\right)$ as well as from the LHC Higgs searches $(g g \rightarrow A \rightarrow \tau \tau$ or $g g \rightarrow H \rightarrow \tau \tau)$. Fig. 1 b shows the allowed $\zeta_{u}$ and $\zeta_{d}$ regions by $B$-decays. The green space indicates the parameter range allowed by both decay modes. It shows that $\zeta_{u}$ is more restricted compared to $\zeta_{d}$. $\zeta_{u}$ is also constrained by LHC Higgs searches. Large $\zeta_{u}$ produces abundant intermediate Higgs $H$ and consequently results in excessive $\tau$ final states. Fig. 1c shows $\zeta_{u}$ upper bounds allowed by $B$-decays and LHC. Depending on the Higgs boson masses and $\zeta_{l}$, the upper bounds on $\zeta_{u}$ are determined by $B$-physics or by LHC results. The overall combined upper bound of $\zeta_{u}$ lies between 0.3 and 0.6.

The LHC upper limit on $\zeta_{u}$ is also related to the triple Higgs coupling constant $C_{H A A}$. Large $C_{H A A}$ which is strongly correlated with $C_{H_{H^{+} H^{-}}}$enhances the decay process $H \rightarrow A A$. Thus the final $\tau$ states are regulated by either $\zeta_{u}$ or $C_{H^{+} H^{-}}$. Fig. 2a shows the proportional relation between $\mathrm{C}_{\mathrm{HH}^{+} \mathrm{H}^{-}}$and the possible maximum of $\zeta_{u}$. It also shows the upper limit of $\mathrm{C}_{\mathrm{HH}^{+} \mathrm{H}^{-}}$, which is constrained by theoretical and electro-weak constraints. The upper limit of $C_{H_{H}+H^{-}}$determines the maximum of the bosonic contributions $a_{\mu}^{B} 2 \mathrm{HDM}$ along with $\zeta_{l}$.

\section{The overall maximum of the muon $g-2$ and conclusion}

Figs. $2 \mathrm{~b}$ and $2 \mathrm{c}$ show the possible range of $a_{\mu}^{2 \mathrm{HDM}}$ for different $\zeta_{l}$ and $M_{H}=M_{H^{ \pm}}$. The yellow 


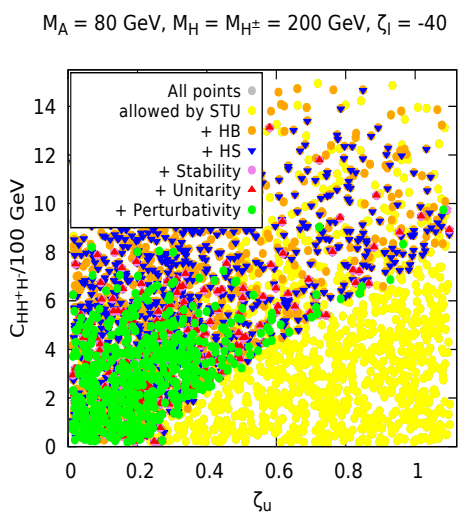

(a)

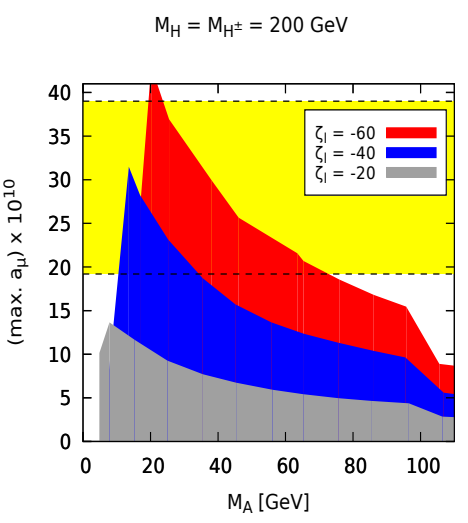

(b)

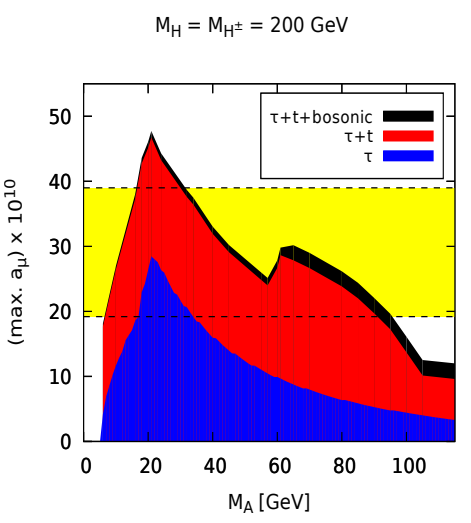

(c)

Figure 2

band indicates the current discrepancy in the muon $g-2$ in Eq. 1.1. The plot in Fig. 2b shows $a_{\mu}^{2 \mathrm{HDM}}$ for different $\zeta_{l}$. For fixed $M_{A}$ and $\zeta_{l}$ the maximum of $\zeta_{u}$ obtained in Fig. 1c is adopted. We need $\left|\zeta_{l}\right|>30$ to explain the discrepancy as we cannot find any $M_{A}$ points to lie in the yellow band for $\zeta_{l}=-20$ (the gray region). Fig. 2c shows the overall maximum of $a_{\mu}^{2 \mathrm{HDM}}$ in the 2HDM. The allowed maxima of $\zeta_{l}$ and $\zeta_{u}$ for given Higgs masses are used to evaluate the possible maximum of $a_{\mu}^{2 \mathrm{HDM}}$. The blue space is only from the $\tau$-loop, the red space from adding the $t$-loop. The black space indicates the bosonic corrections. We observe that the maximum of $a_{\mu}^{2 \mathrm{HDM}}$ reaches up to $\sim 45 \times 10^{-10}$ around $M_{A}=20 \mathrm{GeV}$ and that it is possible to explain the discrepancy at low mass scales $20<M_{A}<100 \mathrm{GeV}$ in the $2 \mathrm{HDM}$ in contrast to the Minimal Supersymmetric SM (MSSM), where the typical scales are $M_{\mathrm{NP}} \sim 500 \mathrm{GeV}$ or $M_{\mathrm{NP}} \sim 1 \mathrm{TeV}$ for the scenario with $\tan \beta \rightarrow \infty$ [8]. This result further motivates searches of low scale pseudoscalar Higgs bosons at the LHC.

\section{References}

[1] A. Cherchiglia, D. Stöckinger and H. Stöckinger-Kim, Phys. Rev. D 98 (2018) 035001 [arXiv:1711.11567 [hep-ph]].

[2] A. Cherchiglia, P. Kneschke, D. Stöckinger and H. Stöckinger-Kim, JHEP 1701 (2017) 007 [arXiv:1607.06292 [hep-ph]].

[3] G.W. Bennett, et al., (Muon ( $g-2)$ Collaboration), Phys. Rev. D 73, 072003 (2006).

[4] T. Aoyama, M. Hayakawa, T. Kinoshita and M. Nio, Phys. Rev. Lett. 109, 111808 (2012) [arXiv:1205.5370 [hep-ph]].

[5] C. Gnendiger, D. Stöckinger and H. Stöckinger-Kim, Phys. Rev. D 88, 053005 (2013) [arXiv:1306.5546 [hep-ph]].

[6] M. Davier, A. Hoecker, B. Malaescu and Z. Zhang, Eur. Phys. J. C 77 (2017) no.12, 827 [arXiv:1706.09436 [hep-ph] ]; A. Keshavarzi, D. Nomura and T. Teubner, Phys. Rev. D 97 (2018) no.11, 114025 [arXiv: 1802.02995 [hep-ph]].

[7] A. Pich and P. Tuzon, Phys. Rev. D 80, 091702 (2009) [arXiv: 0908.1554 [hep-ph] ].

[8] M. Bach, J. h. Park, D. Stöckinger and H. Stöckinger-Kim, JHEP 1510 (2015) 026 [arXiv:1504.05500 [hep-ph]]. 